\title{
Root resection: an alternative treatment option to extraction
}

\begin{abstract}
An 80 year old male patient was referred to restorative postgraduate waiting list for replacement of missing maxillary posterior teeth affecting masticatory function. On further examination right maxillary second molar was diagnosed with vertical root fracture. The mesiobuccal root was resected and subsequently following healing a milled metal crown was cemented. The missing teeth were replaced with a removable partial overdenture.
\end{abstract}

Keywords: root resection, vertical root fracture, removable partial overdenture, milled crown
Volume 6 Issue 5 - 2017

\author{
Asfand Ali Khan, Raja Noreihan, Hadijah \\ Abdullah \\ Department of Restorative Dentistry, University of Malaya, \\ Malaysia
}

Correspondence: Asfand Ali Khan, Department of Restorative Dentistry, Faculty of Dentistry, University of Malaya, 50603, Kuala Lumpur, Malaysia, Tel 60-16-2597259,

Email masfandali@um.edu.my

Received: September 20, 2016 | Published: March 07, 2017

\section{Introduction}

Root resection is the process by which one or more of the roots of a tooth are removed at the level of the furcation while leaving the crown and remaining roots in function. ${ }^{1}$ Indications for root resection therapy include: teeth with periodontal problems, endodontic problems, root fractures, and prosthetic problems. ${ }^{2}$ Vertical root fracture (VRF) is an untoward complication to root canal therapy that often calls for tooth extraction. Aetiological factors for VRF include loss of substance due to restorative and endodontic therapy and stress factors associated with root canal debridement and filling. ${ }^{3}$ Root canal preparation causes stress in dentin and may initiate dentinal cracks, which can extend to complete fractures under masticatory forces. ${ }^{4} \mathrm{VRF}$ usually originates from the apical end of the root and propagates coronally or depending on the nature of stresses, originate from the cervical portion of the root with extension in an apical direction. ${ }^{4}$ Usually, patients of middle and older age are affected. An overall prevalence of 3\% to 5\% has been reported in literature. ${ }^{5}$ The clinical presentation of VRF is extremely variable and may include fistula and abscess formation, development of a deep, narrow and isolated pocket, radiographically visible bone resorption along the lateral surface of the root and depending the on the angle of the X-ray cone beam, radiographically visible fracture line. ${ }^{6}$

\section{Case report}

An 80 year old male patient was referred to restorative postgraduate waiting list for replacement of missing posterior maxillary teeth. His chief complaint was that he could not chew food properly because of his missing back teeth. Patient had complicated past medical history of Coronary Artery Disease, Atrial fibrillation and Asthma. Patient also had a habit of clenching his teeth subconsciously which he was not aware of. On extra-oral examination patient had bulky masseter muscles and class III skeletal profile (Figure 1). On intra-oral examination he had missing right maxillary second premolar, right maxillary first molar and left maxillary first molar. The retained root of right maxillary first premolar was present (Figure 2). The maxillary right second molar had a large amalgam filling and was root treated (Figure 3). The amalgam filling dislodged while the patient was under treatment. The remaining tooth structure was assessed and no crack line was noted. The tooth was immediately restored with composite restoration. 6-point periodontal probing was also performed and the maximum probing depth was $4 \mathrm{~mm}$ at the mesio-buccal (MB) line angle. After crown preparation was done, a crack became visible at the MB line angle. The pocket depth was noted to be $5 \mathrm{~mm}$. The pocket was narrow and isolated. During cord packing there was discharge of pus and blood from the pocket. Temporary crown was cemented as the patient was travelling overseas for 2 months. The tooth was reassessed upon patient's return and the probing depth at MB line angle had increased to $6 \mathrm{~mm}$ and pus discharge was noted. Periapical radiograph was taken which showed crack line along the MB root (Figure 4) therefore diagnosis of VRF was made. The following treatment options for replacing the missing maxillary where discussed with the patient:

1. Extraction of fractured tooth followed by removable partial overdenture.

2. Extraction of fractured tooth followed by implant supported prosthesis.

3. Root resection of $\mathrm{MB}$ root followed by removable partial overdenture.

After considering patient's complex medical history for implant placement surgery and loss of retention \& support for removable partial overdenture following extraction of maxillary second molar (the last tooth in the quadrant), the treatment option of root resection and tooth supported cobalt chromium overdenture was selected. Prior to commencement of surgical procedure of root resection, the patient's cardiologist was consulted as patient was taking an anticoagulant Dabigatran $110 \mathrm{mg}$ twice daily. The patient was advised to stop taking Dabigatran 24 hours prior to the surgical procedure and restart intake the evening after surgery once the wound had stabilized and bleeding had stopped. The surgical procedure was performed 
by Dr. Raja Noreihan. The tooth structure coronal to the MB root was removed to outline the MB root for easy root resectioning and visualise the extent of the crack. The surgical area was anaesthetised and an envelope muco-periosteal (full thickness flap) was raised. The $\mathrm{MB}$ root was resectioned using a diamond fissure bur and removed with a root extraction forcep. The socket was irrigated with saline and cleaned using a curette. Calcium sulphate chips were packed in the socket. Surgical sponge was placed on the chips and the flap was sutured using Mersilk® 4.0 sutures. The sutures were removed 10 days later and the tooth was reviewed after 1 month (Figure 5). Crown preparation on maxillary second molar for milled metal crown was done 10 weeks after root resection surgery (Figure 6). A milled metal ceramic crown was placed on maxillary left second premolar and a metal coping on maxillary right first premolar (retained root). A cobalt chromium removable partial overdenture with rest seats on right maxillary second molar, right canine, left second premolar and left second molar was fabricated and issued (Figure 7).

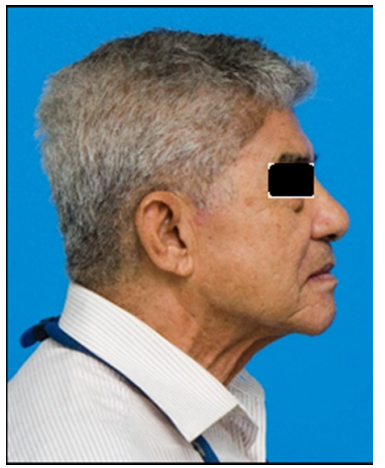

Figure I Right profile view.

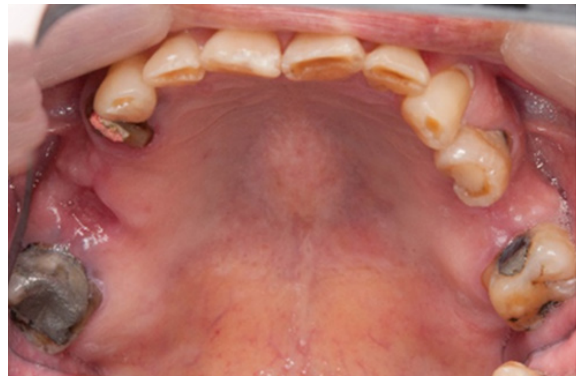

Figure 2 Preoperative intra oral view of maxillary arch.

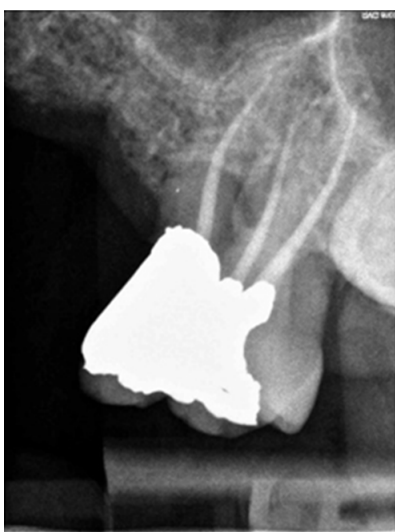

Figure 3 Preoperative periapical radiograph of maxillary right second molar.

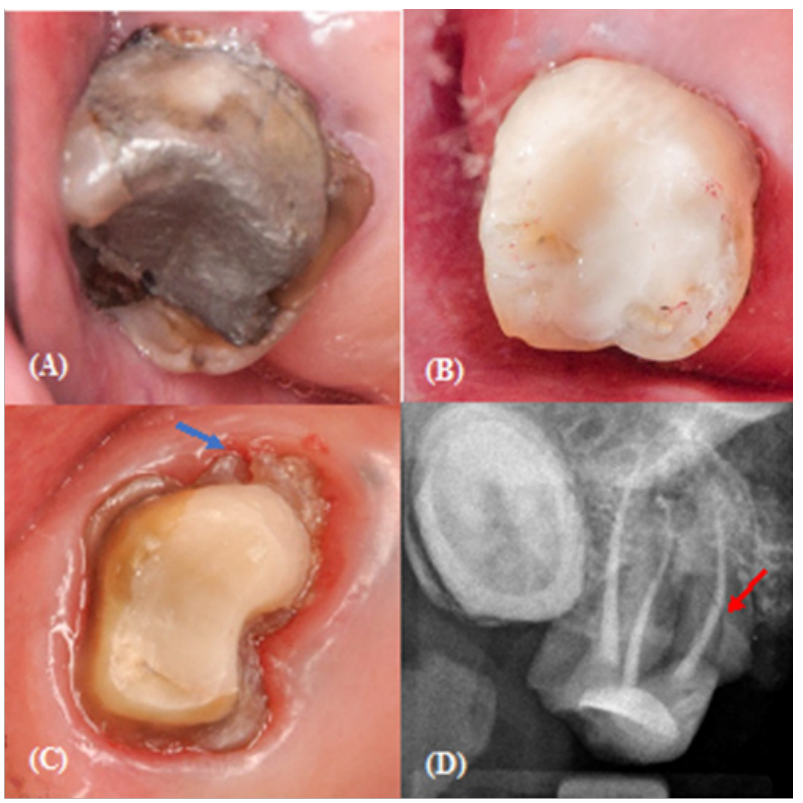

Figure 4 Cracked tooth assessment, A: Preoperative photograph; B: Post composite restoration; C: Post crown preparation (Crack visible - blue arrow); D: Radiograph post crown preparation (Crack line visible -red arrow).

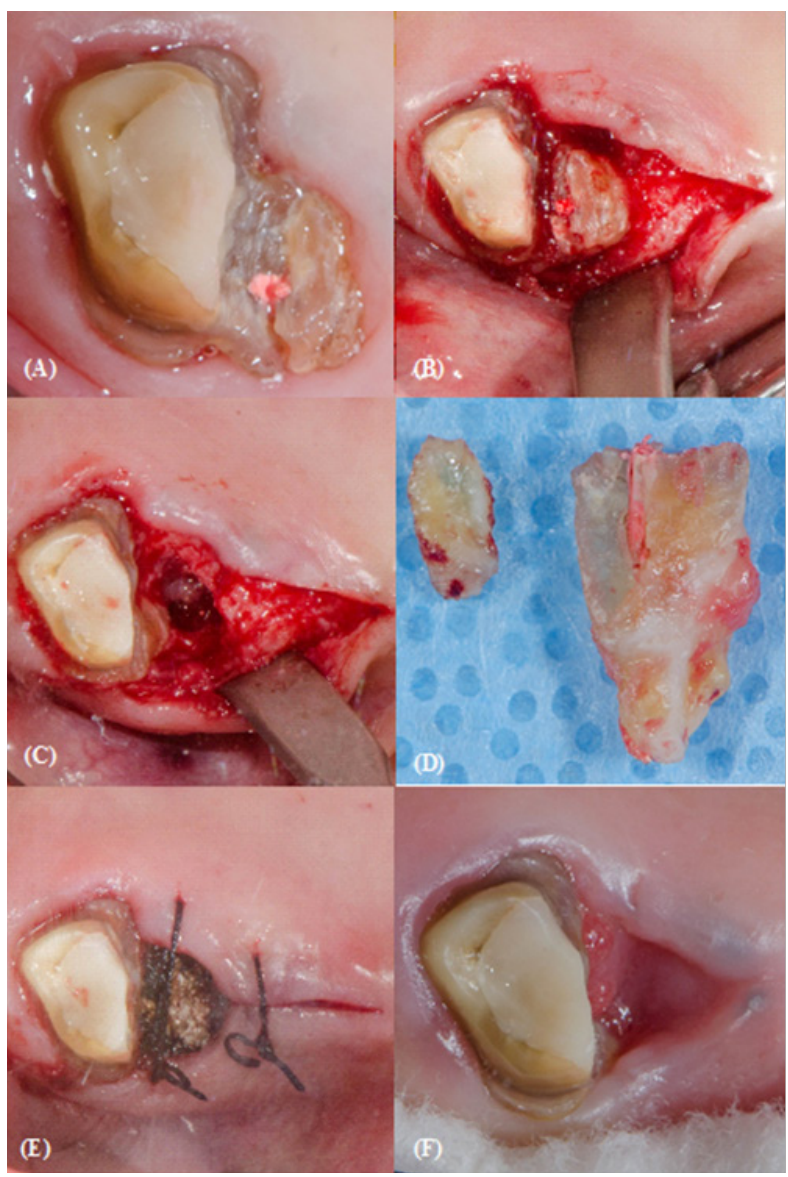

Figure $5 \mathrm{~A}$ :After removal of tooth structure coronal to $\mathrm{MB}$ root, the crack is visible. $B: M B$ root resectioned from the tooth. C: Socket post removal of $M B$ root; D: Sectioned MB root with fractured fragment; E: At suture placement; F:At I month review. 


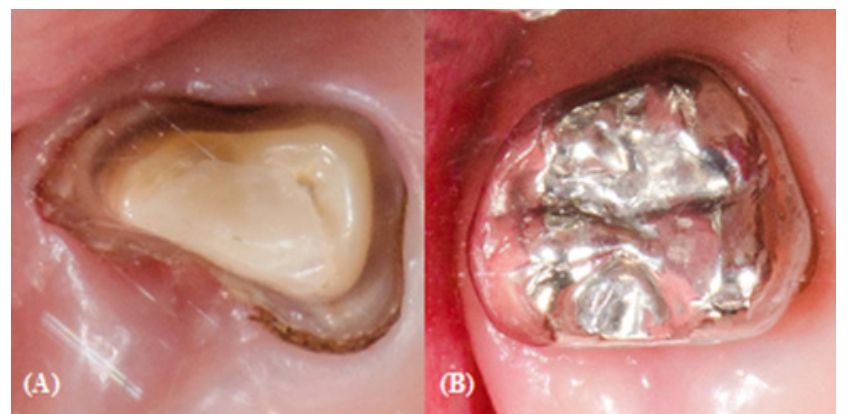

Figure 6 A: Crown preparation 10 weeks after root resection; B: Cemented milled metal crown.

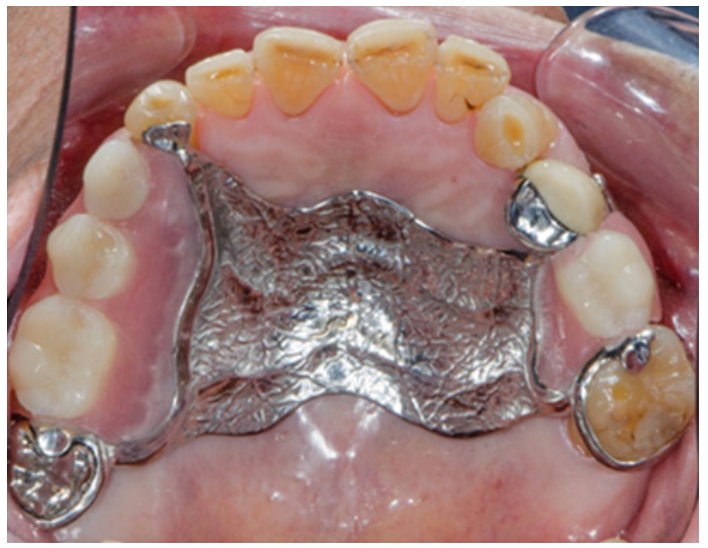

Figure 7 Postoperative photograph of maxillary arch.

\section{Discussion}

Treatment of vertically fractured root is dependent on the tooth type as well as extent, duration and location of fracture. Multi-rooted teeth can often be successfully treated by root resection. Because root resection is very technique sensitive and complex, proper case selection is essential. ${ }^{7}$ In this case there was sufficient bone support around the remaining roots and sufficient coronal tooth structure as well, therefore, the treatment option of root resection followed by milled metal crown for providing support to the removable partial overdenture was selected. Prognosis for root resected posterior teeth is good provided the fracture can be removed in its entity. The retention rate of root resected teeth at 10 years has been reported at $70.2 \%{ }^{8}$ Some investigators have compared the prognosis of root resection therapy to that of implant therapy. Fugazzotto ${ }^{9}$ reported 15-year cumulative success rates of $96.8 \%$ for root resected molars and $97.0 \%$ for molar implants. He concluded that molar root-resection therapy and implant therapy had a high degree of functional success. Conventional tooth-retained overdentures are a simple and cost effective treatment option. When the tooth is firm but compromised, it can be retained and used as abutment for overdenture fabrication. Advantages of overdentures include preservation of alveolar bone, proprioception and enhanced stability and retention. One significant disadvantage of overdentures is that meticulous oral hygiene is necessary to prevent caries and periodontal disease. ${ }^{10} \mathrm{~A}$ longitudinal study that followed up some patients up to 22 years reported rate of abutment tooth loss under overdentures at $20 \%$ with periodontal disease followed caries as being the most common causes. They concluded that failure of many abutment teeth could have been prevented if patients had practiced better oral hygiene. ${ }^{11}$ Masticatory function was the main reason for which the patient came for treatment and his main concern was addressed. The healing around maxillary right second molar was satisfactory and the prognosis is deemed to be good.

\section{Funding}

None.

\section{Acknowledgements}

None.

\section{Conflicts of interest}

The authors declare that there is no conflict of interest.

\section{References}

1. Newell DH. The role of the prosthodontist in restoring root-resected molars: a study of 70 molar root resections. J Prosthet Dent. 1991;65(1):715 .

2. Basaraba N. Root amputation and tooth hemisection. Dent Clin North Am. 1969;13(1):121-132.

3. Haueisen H, Gartner K, Kaiser L, et al. Vertical root fracture: prevalence, etiology, and diagnosis. Quintessence Int. 2013;44(7): 467-474.

4. Tamse A. Vertical root fractures in endodontically treated teeth: diagnostic signs and clinical management. Endodontic topics. 2006;13(1):84-94.

5. Roh BD, Lee YE. Analysis of 154 cases of teeth with cracks. Dent Traumatol. 2006;22(3):118-123.

6. Moule AJ, Kahler B. Diagnosis and management of teeth with vertical root fractures. Aust Dent J. 1999;44(2):75-87.

7. De Sanctis M, Murphy KG. The role of resective periodontal surgery in the treatment of furcation defects. Periodontol. 2000;22: 154-168.

8. Park SY, Shin SY, Yang SM, et al. Factors influencing the outcome of rootresection therapy in molars: a 10-year retrospective study. $J$ Periodontol. 2009;80(1):32-40.

9. Fugazzotto PA. A comparison of the success of root resected molars and molar position implants in function in a private practice: results of up to 15-plus years. J Periodontol. 2001;72(8):1113-1123.

10. Thayer HH. Overdentures and the periodontium. Dent Clin North Am. 1980;24(2):369-377.

11. Ettinger RL, Qian F. Abutment tooth loss in patients with overdentures. $J$ Am Dent Assoc. 2004;135(6):739-796. 